\title{
Enquête
}

Archives de la revue Enquête

\section{2| 1985}

\section{Au fil de la lignée}

\section{Annexes}

\section{(2) OpenEdition}

1 Journals

Édition électronique

URL : http://journals.openedition.org/enquete/235

DOI : 10.4000/enquete. 235

ISSN : 1953-809X

Éditeur :

Cercom, Éditions Parenthèses

Édition imprimée

Date de publication : 1 juin 1985

\section{Référence électronique}

«Annexes », Enquête [En ligne], 2 | 1985, mis en ligne le 03 juin 2013, consulté le 30 avril 2019. URL

http://journals.openedition.org/enquete/235; DOI : 10.4000/enquete.235

Ce document a été généré automatiquement le 30 avril 2019 


\section{Annexes}

\section{Note méthodologique}

\subsection{Interviews réalisées lors de la phase de pré-enquête (mars-juin} 1982)

- Direction de la Solmer: M.GATTEGNO, chef du service Mouvement du personnel: M. POULET, chef du service de Formation générale, M. SERPENTIER, Directeur administratif, M. VALERY et M. DAVID, service Information.

- Syndicat CFDT : M. HURIAUX, secrétaire du comité d'établissement, M. LAIRANDEAUX.

- Syndicats CGT et UFICT CGT : M. CHEVALLIER, M. AMIEL, M. TOZIN, M. ZIMMERMANN.

- Municipalité de St-Martin de Crau : M. MIALOC, délégué à l'urbanisme.

- Municipalité d'Istres : M. GAUTHIER, Délégué à Information.

- Municipalité de Martigues : Mme GIRARD, Déléguée à l'urbanisme.

- Municipalité de Miramas : M. VIAL, Secrétaire général adjoint.

- Municipalité de Port-de-Bouc : M. BAEZA, Secrétaire général.

- Lycée d'Enseignement Professionnel Jean-Moulin de Port de Bouc : M. COLLET, Directeur.

\subsection{La construction de l'échantillon et les conditions d'accès aux familles}

1 La population concernée par l'enquête, celle des Familles des travailleurs employés par la Solmer à Fos-sur-Mer, se caractérise par son importance numérique (sans doute environ sept mille familles si l'on y inclut celles des sous-traitants et intérimaires, du site) et son extrême diversité d'origines et de situations professionnelles et résidentielles ${ }^{1}$. De même que pour l'ensemble de l'enquête la recherche d'une quelconque représentativité de l'échantillon étant étrangère à nos objectifs, nous avons constitué notre échantillon à partir du repérage de certains des enjeux sociaux paraissant déterminants. L'appartenance commune des salariés à un (ou deux) collectifs concrets de travail n'a pas été retenue tant pour des raisons d'option théorique - la pertinence d'un tel critère quant à la structuration des modes de vie du personnel d'une telle usine paraissant a priori contestable - que pour des raisons pratiques - impossibilité de déterminer le ou les 
secteurs éventuellement pertinents sans mobiliser des démarches d'enquête lourdes et spécifiques.

2 C'est ainsi que la prise en compte de quatre types d'enjeux nous paraissant clefs a amené à retenir quatre principaux critères :

- le rôle croissant des catégories intermédiaires de salariés, situés sur des lignes de fractures de rapports sociaux a déterminé le choix des catégories de techniciens, d'agents de maîtrise et de « spéciaux »;

- la structure d'âge du personnel a permis de retenir des tranches d'âge à la fois suffisamment homogènes pour saisir la manière dont les individus font face à des changements intervenant pour la plupart d'entre eux au cœur de leur cycle de vie professionnelle, et à la fois suffisamment diverses pour saisir des effets de génération. Les tranches d'âge suivantes ont donc été retenues : 30 à 40 ans pour les « spéciaux » et les techniciens, 35 à 55 ans pour les agents de maitrise ;

- pour éviter une trop grande dispersion des situations résidentielles des familles, tout en prenant en compte le mouvement d'éclatement de l'espace de résidence vers les zones pavillonnaires éloignées du site industriel, nous avons retenu d'une part cinq des plus importantes communes de résidence des salariés (St-Martin de Crau, Istres-Entressen, Martigues, Miramas) et d'autre part un petit nombre de villages ;

- enfin nous souhaitons pouvoir rencontrer quelques familles dans lesquelles les deux membres du couple travaillaient chez Solmer, situation relativement fréquente notamment chez les technicien(ne)s et les employé(e)s.

3 Les 45 familles rencontrées ont été contactées par correspondance ${ }^{2}$, à partir d'un échantillon par quotas constitué à partir des critères ci-dessus. Nous avons contacté au total 200 ménages. Le taux d'acceptation, globalement faible (environ $20 \%$ ) s'explique par le type de démarche utilisée.

4 Le renvoi d'une lettre supposait une motivation relativement forte pour l'enquête, sans pour autant que le sens ait pu en être explicité a posteriori de manière simple: On note simplement dans notre échantillon une légère sur-représentation des syndiqués et exsyndiqués.

5 Une relance systématique au domicile même auprès d'une partie des "spéciaux» contactés par courrier, catégorie pour laquelle le taux de réponse était sensiblement plus faible, a permis d'obtenir environ la moitié de réponses positives, l'autre moitié refusant de nous recevoir. L'importance de ce taux de refus nous semble liée au moins pour une part aux fortes tensions dans les rapports sociaux à l'usine ${ }^{3}$.

6 L'échantillon des 45 familles se caractérise finalement comme suit :

Communes de résidence des ménages selon la catégorie professionnelle de l'homme

\begin{tabular}{|c|c|c|c|c|c|c|c|}
\hline 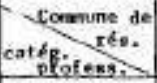 & $\begin{array}{c}\text { 19TRES } \\
\text { ENTRRSSPN }\end{array}$ & $\begin{array}{c}\text { SE, MARTIK } \\
\text { OE CRAU }\end{array}$ & $\begin{array}{l}\text { marti- } \\
\text { cans }\end{array}$ & MIRNUS & $\begin{array}{l}\text { POAT DF } \\
\text { BOUK: }\end{array}$ & Autres & tots 1 \\
\hline OP1, op2 & 1 & & 1 & & & 1 & 3 \\
\hline Spécial & 2 & & 1 & 2 & 5 & 3 & 13 \\
\hline $\begin{array}{l}\text { Regent de } \\
\text { materine }\end{array}$ & 5 & 6 & 2 & 1 & 1 & 2 & 17 \\
\hline $\begin{array}{l}\text { Tectini: } \\
\text { eiens }\end{array}$ & 3 & 3 & 1 & 1 & & 4 & 12 \\
\hline TOTN, & 11 & 9 & 5 & 4 & 6 & 10 & 45 \\
\hline
\end{tabular}


Tranche d'âge des salariés selon la catégorie professionnelle

\begin{tabular}{|c|c|c|c|c|c|c|}
\hline $\begin{array}{c}\text { Canachog } \\
\text { eaces. } \\
\text { profens. }\end{array}$ & $<30$ & 31 a 35 & $36 \div 50$ & $\begin{array}{l}\text { eotn1 } \\
30 \& 40\end{array}$ & $>41$ & TOTNS. \\
\hline OP1, OP2 & 3 & & & & & 3 \\
\hline spécial & 2 & 1 & 3 & 10 & , & 13 \\
\hline $\begin{array}{l}\text { Agenti } \\
\text { de naltrise }\end{array}$ & & ' & $B$ & 9 & B & 17 \\
\hline $\begin{array}{l}\text { Techni- } \\
\text { eienn }\end{array}$ & 1 & $" 1$ & & 11 & , & 16 \\
\hline Emplaye es & & 2 & & & , & 3 \\
\hline TOTAL. & B & 20 & $" 1$ & 31 & 13 & so \\
\hline
\end{tabular}

soit :

Catégorie professionnelle de l'homme

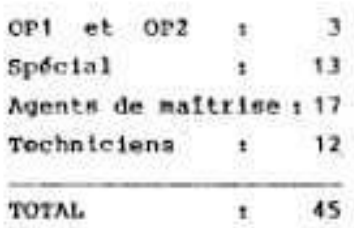

Commune de résidence des familles

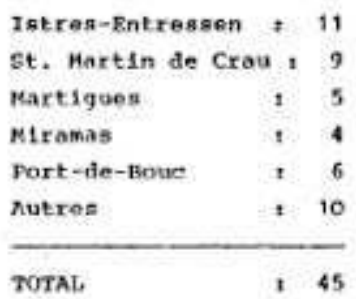

Tranches d'âge des salariés

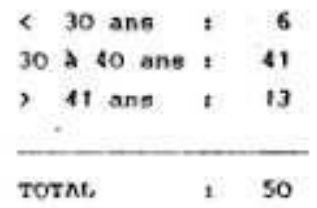

75 ménages dont les deux membres travaillent chez Solmer : 2 où l'homme est technicien et la femme technicienne ; 3 où l'homme est technicien et la femme employée.

8 La quasi-totalité des interviews ont été réalisées en présence conjointe de l'homme et de la femme. 


\subsection{L'Analyse des entretiens et le choix des cas des familles dont les biographies guident l'exposé des résultats}

9 Outre la mise en œuvre de la méthodologie commune aux quatre équipes quant au guide d'entretien et au traitement des données biographiques en résultant, on a effectué un travail de positionnement - par rapprochement ou opposition - des individus et des familles dans l'espace social et ses diverses dimensions: trajectoires sociales, cursus professionnel, organisation du cycle de vie, structure des champs éthico-moraux - tout particulièrement du point de vue du rapport au travail et à l'identité sociale de classe. Au sein des cas se révélant ainsi les plus pertinents c'est l'inscription dans les processus sociaux les plus caractéristiques de la situation industrialo-urbaine étudiée ainsi que la richesse même de l'entretien qui ont permis d'opérer les choix.

10 La rédaction du texte s'est ainsi efforcée, non pas de rendre compte de manière synthétique de biographies familiales singulières saisies en elle-même, mais d'éclairer à l'aide de ces biographies à la fois une question de portée plus générale (le passage à la maîtrise, le transfert des anciens Lorrains, le passage du chantier à l'usine, la prise de distance résidentielle vis-à-vis de l'entreprise...) et d'autres aspects transversaux aux familles rencontrées de « comme on fait sa vie »...

\section{Données sur le personnel Solmer}

\subsection{La grille des classifications}


(Annexe au protocole du 10 mai 1976)

- Zone de coefficients pour les catégories Ouvriers, Techniciens et Agents de maîtrise

- Positionnement du coefficient d'embauche selon le diplôme obtenu

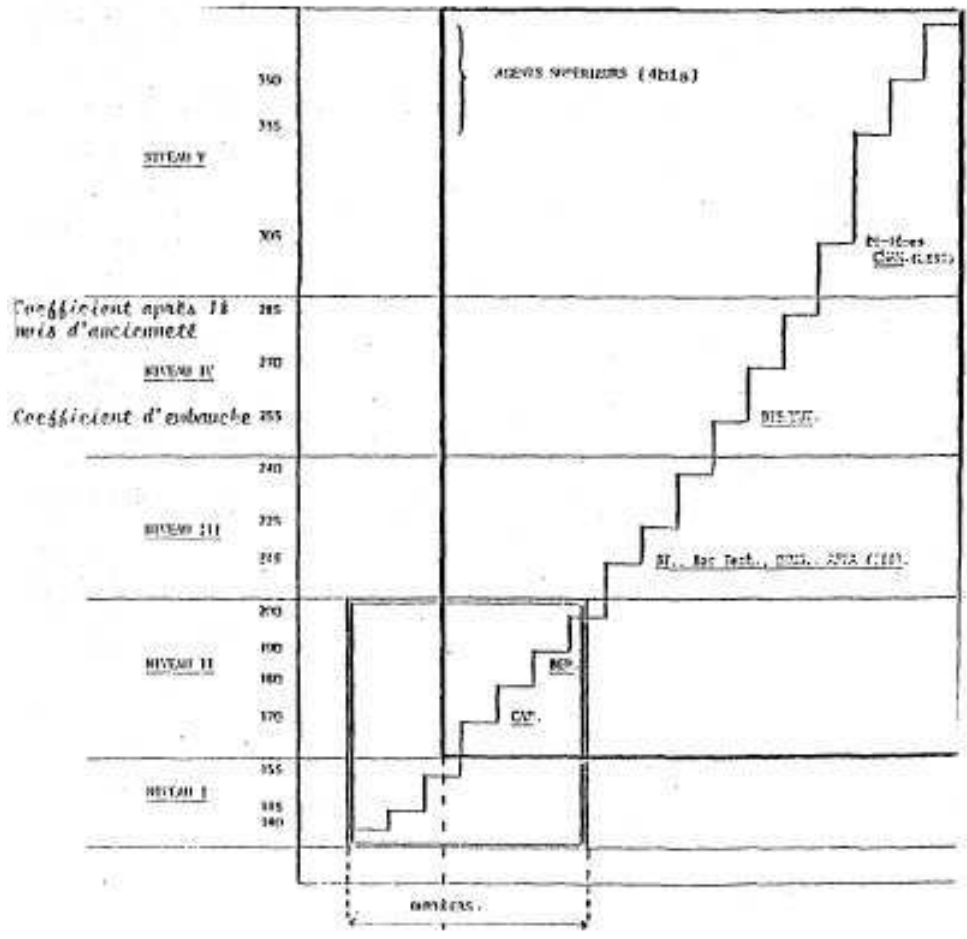

\subsection{Salaires du personnel, revenus des familles}

Rémunération mensuelle moyenne versée en 1982 aux salariés Solmer

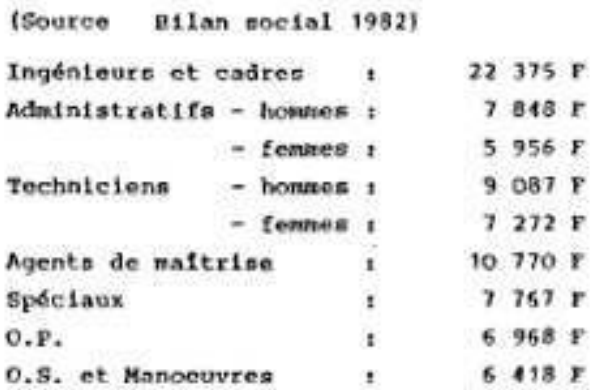

Revenus mensuels déclarés par les ménages lors de l'entretien, selon la catégorie du mari

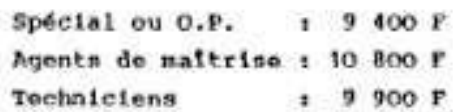

11 La majorité des techniciens sont en horaire de jour et ne touchent donc pas l'importante prime des postes en $3 / 8$. 
2.3. L'influence des confédérations syndicales en 1975 et en 1984

Résultats des élections des délégués du personnel en 1975 et 1984

\begin{tabular}{|c|c|c|c|c|c|c|}
\hline & İe eol- & $\begin{array}{l}975 \\
2^{*} \text { eol-1- } \\
\text { 12ege }\end{array}$ & $\begin{array}{l}\text { jensen- } \\
\text { ble }\end{array}$ & $\begin{array}{l}1_{\text {iege }} \text { col- } \\
\text { ieg }\end{array}$ & $\begin{array}{c}1984 \\
2^{*} \mathrm{col}-1 \\
189 \mathrm{ge}\end{array} \mid$ & $\begin{array}{l}\text { ansen - } \\
\text { ble }\end{array}$ \\
\hline Inucrite & $\begin{array}{r}3855 \\
\text { (1) }\end{array}$ & 2650 & 6505 & 2839 & 3307 & 6146 \\
\hline Expe Intir & $\begin{array}{l}2509 \\
65,0\end{array}$ & $\begin{array}{l}1830 \\
69,1\end{array}$ & $\begin{array}{l}4339 \\
66,7\end{array}$ & $\begin{array}{l}1853 \\
65,3\end{array}$ & $\begin{array}{c}2169 \\
55\end{array}$ & $\begin{array}{l}1002 \\
65,1\end{array}$ \\
\hline$C \in T$ & 47,1 & 17,4 & 34,6 & 39,7 & 14.4 & 26,1 \\
\hline$C F D T$ & 42,0 & 52,3 & 16,3 & 37 & 33,1 & 32,2 \\
\hline r.o. & 10.7 & 30,3 & 19,0 & 16,8 & 18,7 & 17,6 \\
\hline CF TC & & & & & 4,0 & 1,8 \\
\hline c a c & & & & & 24,8 & 13,3 \\
\hline
\end{tabular}

1. Une partie des «Spéciaux », classés ETAM, votent dans le collège Ouvriers.

\subsection{Structure d'âge par catégories}

(cf. Annexe infra)

\subsection{Situations familiales par catégories}

(cf. Annexe infra)

\section{Données sur l'échantillon}

\subsection{Arbres généalogiques des sept familles}

Les Nouge

Les Tocela

Les Rainard

Les Mondan

Les Illico

Les Orion 
Les Constantin

\subsection{Trajectoires professionnelles des 50 salariés en fonction de trois grands types d'origines professionnelles}

(cf. Annexes infra)

\subsection{Trajectoires familiales des 45 ménages : autres données}

(cf. Annexes infra)

\section{NOTES}

1. Sur ces deux points seuls des trois autres situations industrialo-urbaines étudiées, le « terrain » grenoblois est comparable à celui de Fos. Encore que l'espace de résidence des salariés de Merlin-Gerin soit sensiblement plus restreint que celui de la zone de Fos et de l'Étang de Berre.

2. 5 de ces familles ont été contactées ultérieurement par l'intermédiaire des ménages rencontrés : il s'agit de parents s'avérant travailler également chez Solmer.

3. Fait significatif de ces tensions (au-delà d'un impact probablement faible quant au taux de réponses favorables), la direction de l'usine a largement diffusé, au moment de notre enquête, l'information selon laquelle elle ne la cautionnait en aucune manière. 